\title{
Development of an ETSI Standard for Phase-2 GSM/DCS Mobile Terminal Conformance Testing
}

\author{
Hu., Wattelet. and Wang.* \\ ETSI \\ Route des Lucioles, 06921 Sophia Antipolis CEDEX, France \\ +33 49294 4369, +33 49365 3851, shicheng.hu@etsi.fr \\ jean-claude.wattelet@etsi.fr_nwang@cs.ecnu.edu.cn
}

\begin{abstract}
The European Telecommunications Standards Institute (ETSI) is responsible for the production and publication of telecommunications standards in Europe and the marketing of these standards world-wide. The ETSI Special Mobile Group (SMG) is responsible for defining and specifying the GSM (Global System for Mobile communications) and UMTS (Universal Mobile Telecommunications System) standards. One of its Sub-Technical Committees, SMG7, is in charge of the specifications of mobile terminal testing standards. This experiences paper describes one of the major results from SMG7: a first Abstract Test Suite (ATS) developed as an European Telecommunication Standard (ETS) for mobile terminal conformance testing.
\end{abstract}

Keywords

ATS, DCS, ETSI, GSM, mobile, SMG, signalling test, standard, TTCN, validation

\footnotetext{
" Prof. Wang's current address: East China Normal University, Department of Computer Science 3663 N.Zhongshan Rd., 200062 Shanghai, China
} 


\section{GENERAL}

The Phase- $2^{\dagger}$ GSM mobile terminal conformance testing standards are specified in an ETSI standard, ETS 300607 (GSM 11.10), in multiple parts. The testing standard covers the conformance requirements for mobile terminals at the radio interface, the reference point Um, for the frequency bands 900 for GSM and/or $1800 \mathrm{Mhz}$ for DCS. The standard includes radio, speech, Subscriber Identity Module (SIM) testing, the Data Link (L2) and the Network Layer (L3) testing, and different kinds of service-related L3 protocol testing. More than 600 test cases are described and specified in English prose in the standard ETS 300 607-1.

No TTCN specification is available for the Phase-1 testing. In order to use full advantages of the testing methodology described in the ISO 9646, to reduce the costs of signalling (Network protocol) tests and the cost of the tester itself, and to avoid possible ambiguity of test descriptions, and therefore to increase the quality and applicability of the testing standard, it was decided to convert the existing L3 signalling test descriptions in the ETS $300607-1$ into an ATS in TTCN.

The development of the ATS for Phase-2 GSM/DCS mobile terminal conformance testing was started in September 1994. The design of the ATS was undertaken by ETSI project teams which were funded under European Commission Mandates ${ }^{\ddagger}$. The ATS was published by ETSI in September 1996 as ETS 300 607-3. During this period, four ETSI TBRs (Technical Basis for Regulation) TBR19, TBR20, TBR31 and TBR32 were specified by SMG for the technical requirements to be met by mobile terminals capable of connecting to a public GSM/DCS network. A subset of the ATS has been selected as a part of mobile regulatory (approval) testing in Europe. In parallel to the ATS production, a TTCN stand-alone tester was developed by a UK-based company, Anite Systems, under the contract of the GSM Memorandum of Understanding association. Currently, the ETSI GSM ATS is used by 6 European test houses and more than 20 world-wide mobile manufacturers and GSM operators.

\section{ABSTRACT TEST SUITE}

The ATS was developed manually. The Test Suite Structure (TSS) and Test Purposes (TP) of the ATS are identical to those in the prose test specifications. The one-to-one mapping of TSS\&TP ensures that an implementation of the ATS in a simpler test system (stand-alone TTCN tester) can have comparable test

\footnotetext{
$\dagger$ The development of GSM standards and the deployment of the network in terms of facilities available to users were undertaken in two mutually compatible phases: Phase-1 and Phase-2. The Phase-2+ evolves to introduce new technology and features on the Phase-2 basis.

$¥$ The projects were funded under the EC Mandates: BC-T237, BC-T247, BC-T-316 and co-funded by ETSI and EC under the EC mandate BC-T-342.
} 
results with outcomes from an implementation of the prose test specification on a complex test platform (system simulator).

The GSM L3 protocol at the radio interface consists of three sub-layers for peer-to-peer communications: Radio Resource (RR) management functions, Mobility Management (MM) functions and Connect Management functions (CM). The CM sub-layer is composed of Call Control (CC), Short Message Service (SMS) support and Supplementary Services (SS) support. For each entity a corresponding test group tests all elementary procedures belonging to the entity. In addition, a test group called Structured Procedures underlines the testing of the relationships and interworking of procedures between different entities. Invalid and inopportune tests are sensible for radio protocols because of higher error rates. The BIBO group tests the mobile error handling behaviours. The Initial and Idle Mode test groups are similar to the basic interconnection test in the ISO 9646 conventions. An EGSM test group testing the mobile using the extended GSM frequency band. Finally, the General test group is contributed to the basic bearer or tele- service-related general signalling tests.

More than 700 essential conformance requirements have been identified for the mobile signalling testing. In order to reduce the number of tests most test cases have combined TPs containing 2-3 primary TPs on average. Combined TPs are mostly related to the same elementary procedure. Either they share initial test conditions, or they are described in a consecutive manner. The resulting ATS is more compact and has a total of 324 test cases without changing the test coverage. The ATS has 4.3 MB codes in MP form, under which 3\% codes for Overview, $16 \%$ for Declarations, $26 \%$ for Constraints and 55\% for Dynamic parts.

\section{TEST METHOD AND TEST MODEL}

The whole ATS is based on the Distributed test method in an SPyT context. The PCO consists of an L2 SAPI0 and an SAPI3. Different SAPI values indicate the respective data links. All test events are specified in terms of L2 Primitives and L3 PDUs: RR, MM, CC, SMS or SS message units. Because of having sub-layer model, messages are often chaining embedded. An SMS command as application layer PDU is embedded in a short message (SM) transfer PDU which is in turn embedded in an SM relay protocol (RP). The RP PDU is again embedded in an SM control PDU which is then embedded in an L3 SMS message. An another example is that different kinds of SS components in ASN.1 are embedded in a Facility information element of a CC or SS message in the TTCN definitions.

Since Dm control channels are distributed on several types of channels / subchannels in several possible cells, a parameter referred to as logical channel is introduced to all L2 ASP type definitions to ensure correct distributions of the L3 messages on various types of channel. In order to check whether a message from the mobile under test is received in the correct time, another parameter for 
received TDMA (Time Division Multiple Access) frame number is defined as a time stamp in all DL_INDICATION Primitives type definitions, indicating the received first frame number of a received message by the tester.

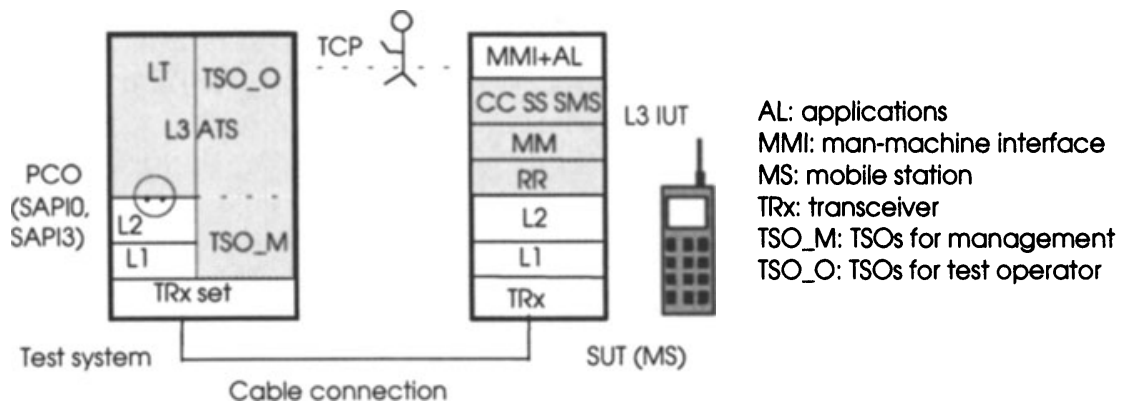

Figure 1 Distributed test method.

Besides the PCO, the ATS needs an additional management interface to communicate with the L2 or L1 of the tester in order to simulate the required GSM network behaviours for testing. The management interface was the most difficult design in the ATS. Effort was made to keep this interface independent of real implementations. Through this interface the ATS is able:

- to configure, deactivate or reactivate a radio channel within a cell;

- to stop a working cell or to generate lower layer failures;

- to manipulate a sending message on a required frame number;

- to prepare for frequency hopping, handover or to down-load a ciphering key;

- to read an L1 header;

- to pre-filter out periodically received measurement reports.

The management interface is specified by defining Test Suite Operations (TSOs). It was a design intention, not to define an additional PCO or a specific set of Primitives for building the management interface, but to keep a clear separation between the well-standardised interface represented by the key word PCO and an interface being standardised in the future version of ISO 9646-3. Nearly 30 TSO_M functions have been specified in the current ATS for this purpose.

To achieve the synchronisation between the (lower) tester and test operator (as upper tester) and management of information exchanges during testing nearly 70 test co-ordination procedures (TCP) are defined as TSOs (TSO_O). 


\section{PRACTICAL AND TECHNICAL CHALLENGES}

It is generally expected within ETSI that an ETSI test suite can be compiled with little human intervention. In order to reach this goal, a series of questions had to be answered in the ATS design phase, for instance:

- how to manage channels;

- how to handle L3 periodic and synchronous signalling;

- how to initialise the tester and the mobile under test;

- how to fill the notation gaps between TTCN and ASN.1;

- how to handle upward compatibility.

\subsection{Channel Management}

Each logical channel needs to be mapped onto a physical channel which has many radio parameters, such as the frequency, time slots, timing advance, etc. to characterise the physical channel. One or more physical channels are mapped onto a radio transceiver. It was a design trade-off as to whether or not the ATS sees transceivers. To avoid over-standardisation, each test case needs to know only which logical channel is currently in use, and what is the physical mapping, and to leave transceivers out of the specification scope.

Furthermore, the stand-alone tester simulates the GSM network functionality at the radio interface. The channel management for each test case has been specified to a certain extent to ensure that test cases can run reasonably.

\subsection{Handling synchronous signalling}

The GSM L3 protocol contains several synchronous signalling. At the downlink (sending) direction System Information (SI) messages are periodically and synchronously broadcast on the Broadcasting Control Channel (BCCH) and on all Slow Associated Control Channels (SACCHs). At the uplink (receiving) direction the tester periodically receives synchronous measurement reports on the SACCHs. However, test events are based on request/ acknowledgement. To extract test events from a synchronous signalling background additional test semantics are defined.

An additional SI buffer is needed in the tester which is capable of storing all SI messages being sent. When an SI message is sent via the PCO, the SI will not be passed to the TTCN out-buffer, but is down-loaded to the SI buffer. As soon as the corresponding $\mathrm{BCCH}$ is configured or a SACCH channel is allocated and initialised, the tester controls the SI buffer sending out the stored SI messages periodically and correctly according to the TDMA timing.

The tester is in the position to prevent the measurement reports from entering the TTCN in-buffer and to provide a control way via the management interface. 


\subsection{Test initial conditions and preambles}

The initial conditions for mobile testing vary to a large extent. It is necessary at the beginning of each test case to bring the mobile under test and the tester itself into pre-defined initial states irrespective of the tester or mobile current state or the SIM contents. This ensures test case independency from each other and is essential when interrupting test execution in the middle of a test, or changing a test execution sequence without changing any of the test results.

Moreover, 25 bearer and tele-services are defined in the Phase-2. It is also necessary before a call establishment to allocate a suitable traffic channel and to prepare an appropriate bearer capability for the service selected for the test.

To cope with these requirements and to avoid specifying a large number of test preambles and constraints for different initial test conditions several general, but highly parameterised test preambles, are designed in the ATS. All initial conditions are presented in terms of a set of independent parameters.

\subsection{Bridging gaps between TTCN and ASN.1}

The SS definitions in ASN.1 came from the GSM Mobile Application Part (MAP) based on the CCITT X.208 (1988) but in addition Ellipsis Notation ("...") is used wherever future MAP protocol extensions are foreseen. All SS specifications in the MAP protocol make use of the Remote Operation Services (ROS) which are served for the exchange of specific SS PDUs' invoking operations and reporting of results or errors. However, the current TTCN does not support:

- the ASN.1 ROS macro definitions in X208 (1988);

- the ASN.1 Ellipsis Notation.

To bridge the notation gaps between ASN.1 and TTCN, all GSM SS used have been redefined in a form which is acceptable by the TTCN and provides the ASN.1 data structures identical to the ROS expansions. The Ellipsis Notation is not tested at all in the ATS. The mobile under test is not allowed to send any protocol extension in the SS protocol.

\subsection{Upward compatibility}

The GSM is one of the fastest moving standardisation areas. When a new type of the Phase- 2 mobile emerges on the market it can already encompass several new Phase-2+ features on top of the Phase- 2 implementation. Thus, the ATS must:

- not fail the mobiles having implemented the new GSM features,

- be easily adapted if adding new features to the existing test cases.

To achieve the upward compatibility nearly 100 ICS and 150 IXIT parameters are defined in the ATS. The corresponding ICS/IXIT questions need to be 
answered by mobile manufacturer. The provided ICS/IXIT parameter values control not only test case selection, but also the selection of an executing path within a test case or a test step, or assigning appropriate values to a constraint according to the features and characteristics of the mobile. For instance, by using a few ICS/IXIT parameters the GSM and DCS mobile tests share the same ATS. Furthermore, the ATS can be also adapted to the PCS testing for mobiles working at the frequency band $1900 \mathrm{Mhz}$.

\section{VALIDATION AND MAINTENANCE}

The ATS has experienced more than one year of validation and maintenance. By exercising executive test cases at the TTCN stand-alone tester the ATS was intensively validated against 10 reference mobile terminals from 4 different sources. The validation results were audited by a third party. Discovered problems were reported to ETSI. A project team was responsible for the ATS maintenance. Based on the problem reports more than 250 change requests (CRs) were produced; 5 versions and 25 revisions of the ATS were delivered. To ensure a minimum quality of each Delivery the ATS was analysed by using 4 different TTCN tools for the syntax and static semantic cross checking. Under all the CRs, $63 \%$ were caused by fixing bugs, $22 \%$ clarified the informally specified TSO_M interface, $15 \%$ followed the changes in the GSM standards.

\section{CONCLUSIONS}

ETSI has developed a complex GSM ATS for GSM/DCS Phase-2 mobile conformance testing within a short period of time. This paper has highlighted the practical and technical challenges of ATS development. Despite the complexity of the GSM standards and various conformance requirements much effort has been made to ensure that the ATS is compilable, feasible, easily adaptable, upward compatible and largely reusable.

The ATS has been validated and is widely used both for mobile regulatory testing and in-house testing. The application of a TTCN specification to mobile terminal testing is now well accepted by the GSM society. However, using TTCN does not necessarily guarantee the correctness of the GSM ATS itself. The validated ATS and the continuous maintenance have added considerable values to the quality of mobiles.

New technology and features in the Phase 2+ GSM standards need to be introduced to the market as fast as possible. Requests have been received to develop new TTCN test specifications for the Phase-2+ mobile testing. The introduction of the ISO 9646-3 edition 2 Mock-Up to ETSI and its implementation in new TTCN tools are essential to meeting the challenge of producing highquality GSM Phase-2+ mobile conformance test specifications. 


\section{REFERENCES}

ETS 300599 (GSM 09.02): "Digital cellular telecommunications system (Phase 2); Mobile Application Part (MAP) specification".

ETS 300 607-1 (GSM 11.10-1): "Digital cellular telecommunications system (Phase 2); Mobile Station (MS) conformance specification; Part 1: Conformance specification".

ETS 300 607-2 (GSM 11.10-2): "Digital cellular telecommunications system (Phase 2); Mobile Station (MS) conformance specification; Part 2: PICS Proforma".

ETS 300 607-3 (GSM 11.10-3): "Digital cellular telecommunications system (Phase 2); Mobile Station (MS) conformance specification; Part 3: ATS".

TBR 19: "European digital cellular telecommunications system; Attachment requirements for Global System for Mobile communications (GSM) mobile stations; Access".

TBR 20: "European digital cellular telecommunications system; Attachment requirements for Global System for Mobile communications (GSM) mobile stations; Telephony".

TBR 31: "European digital cellular telecommunications system; Attachment requirements for mobile stations; in the DCS 1800 band and additional GSM 900 band; Access".

TBR 32: "European digital cellular telecommunications system; Attachment requirements for mobile stations; in the DCS 1800 band and additional GSM 900 band; Telephony".

CCITT Recommendation X.208 (1988): "Specification of Abstract Syntax Notation One (ASN.1)".

\section{BIOGRAPHY}

\section{Shicheng $\mathrm{Hu}$}

Is one of the permanent nucleus at the ETSI PEX competence centre for specification, validation and testing, supports SMG, was the project leader for GSM/DCS ATS, managed as a senior engineer several European testing projects at SIGOS , Germany, worked as a security specialist for Philips.

\section{Jean-Claude Wattelet}

Is a specialist for test and TBR specifications of the ETSI GSM and DECT protocols, maintained GSM/DCS ATS, worked as an engineer for CAP SESA.

Neng Wang

Is a Professor at the East China Normal University for computer networking, communications and protocol testing, developed GSM/DCS ATS, directed a numerous research projects, was a visiting professor at the University of Erlangen, Germany and the University of Illinois, USA. 